\title{
Mirror Neuron Activation Prior to Action Observation in a Predictable Context
}

\author{
Monica Maranesi, ${ }^{1}$ Alessandro Livi, ${ }^{2}$ Leonardo Fogassi, ${ }^{2}$ Giacomo Rizzolatti, ${ }^{1}$ and Luca Bonini ${ }^{1}$ \\ ${ }^{1}$ Istituto Italiano di Tecnologia (IIT), Brain Center for Social and Motor Cognition (BCSMC), 43125 Parma, Italy and ${ }^{2}$ University of Parma, Department of \\ Neuroscience, 43125 Parma, Italy
}

\begin{abstract}
A fundamental capacity of social animals consists in the predictive representation of upcoming events in the outside world, such as the actions of others. Here, we tested the activity of ventral premotor area F5 mirror neurons (MNs) while monkeys observed an experimenter performing (Action condition) or withholding (Inaction condition) a grasping action, which could be predicted on the basis of previously presented auditory instructions. Many of the recorded MNs discharged only during action observation (Action MNs), but one-third also encoded the experimenter's withheld action (Inaction MNs). Interestingly, while most of Action MNs exhibited reactive discharge during action observation, becoming active after the go signal, the majority of Inaction MNs showed predictive discharge. MN population activity as a whole displayed an overall predictive activation pattern, becoming active, on average, $340 \mathrm{~ms}$ before the go signal. Furthermore, MNs became active earlier when the observed action was performed in the monkeys' extrapersonal rather than peripersonal space, suggesting that context-based neural prediction of others' actions plays different roles depending on the monkeys' ability to interact with the observed agent.
\end{abstract}

Key words: area F5; contextual information; motor prediction

\section{Introduction}

Mirror neurons (MNs) are a set of visuomotor neurons first discovered in the ventral premotor area F5 of the macaque that discharge both during the execution and observation of goaldirected motor acts (Gallese et al., 1996; Rizzolatti et al., 1996). Based on this property, it has been proposed that MN activity provides the observer with a first-person motor representation of others' actions, enabling an automatic form of action understanding.

Since the earliest reports, it has been claimed that the visual response of MNs is triggered by the observation of others' motor acts while they are being performed. This view has received convergent support from several neurophysiological studies, indicating that some sensory description of the ongoing movement is always necessary to trigger MN response (Umiltà et al., 2001; Kohler et al., 2002; Fogassi et al., 2005; Maranesi et al., 2013). Maranesi et al. (2013) demonstrated that, at the population level, $\mathrm{MN}$ response to an observed agent's reaching-grasping action starts $\sim 60$ ms after movement onset, supporting the idea that $\mathrm{MNs}$ generate a reactive, sensory-driven representation of observed actions.

\footnotetext{
Received July 3, 2014; revised Sept. 13, 2014; accepted Sept. 16, 2014.

Author contributions: M.M. and L.B. designed research; M.M., A.L., and L.B. performed research; M.M., A.L., and L.B. analyzed data; L.F., G.R., and L.B. wrote the paper.

This work was supported by the Italian Institute of Technology and the European Commission Grant Cogsystem FP7-250013. We thank Marco Bimbi for his technical assistance.

The authors declare no competing financial interests.

Correspondence should be addressed to Luca Bonini, PhD, Istituto Italiano di Tecnologia (IIT), Brain Center for Social and Motor Cognition, Via Volturno 39, 43125 Parma, Italy. E-mail: luca.bonini@unipr.it.

DOI:10.1523/JNEUROSCI.2705-14.2014

Copyright $\odot 2014$ the authors $\quad 0270-6474 / 14 / 3414827-06 \$ 15.00 / 0$
}

However, predicting others' actions constitutes one of the most challenging tasks that animals have to deal with in natural social contexts, and it represents the essential prerequisite to readily prepare an appropriate behavioral reaction during social interactions (Kilner et al., 2007). Human studies have shown that cortical motor areas can activate before observation of others' actions, provided that sufficient contextual cues are available (Kilner et al., 2004). This would suggest that, in a predictable context, MNs should become active before any observed movement onset. However, in all MN studies performed so far, the onset of the action observed by the monkey was largely unpredictable, thus leaving this issue unresolved.

The only exception to this scenario is constituted by our recent study (Bonini et al., 2014b) in which we recorded F5 MN activity while monkeys performed and observed a go/no-go task instructed by auditory cues. Crucially, the same cues the monkeys had learned to use during task execution enabled them to predict if, and when, the experimenter's hand would subsequently move during task observation. Some of the MNs recorded with this paradigm showed the intriguing capacity of discharging not only during action execution and observation, but also when the observed agent, not the monkey, refrained from grasping (Inaction MNs). This finding suggested that Inaction MNs encode representations of potential action at a highly abstract level, that is, both when the action is performed or withheld by an observed agent.

Here, we show that, in a predictable context, F5 MNs can activate a long time in advance of the beginning of the observed action, and this capacity is more evident among Inaction MNs than "classical" Action MNs. We also show that predictive discharge occurs earlier and more frequently when neurons are 


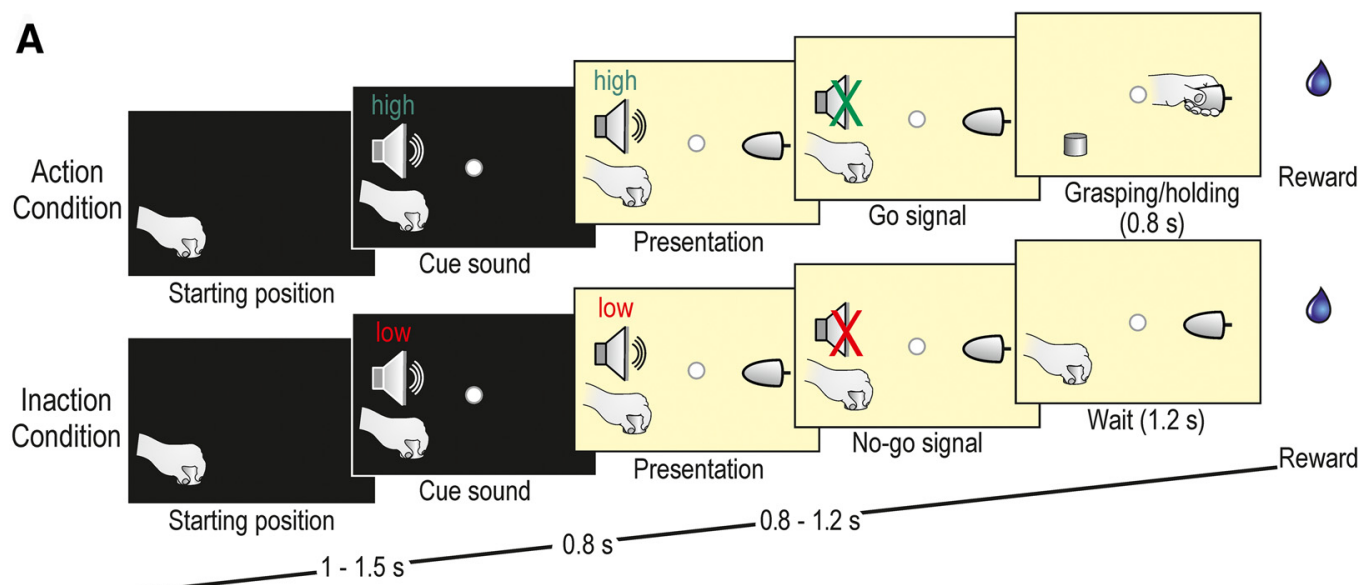

B

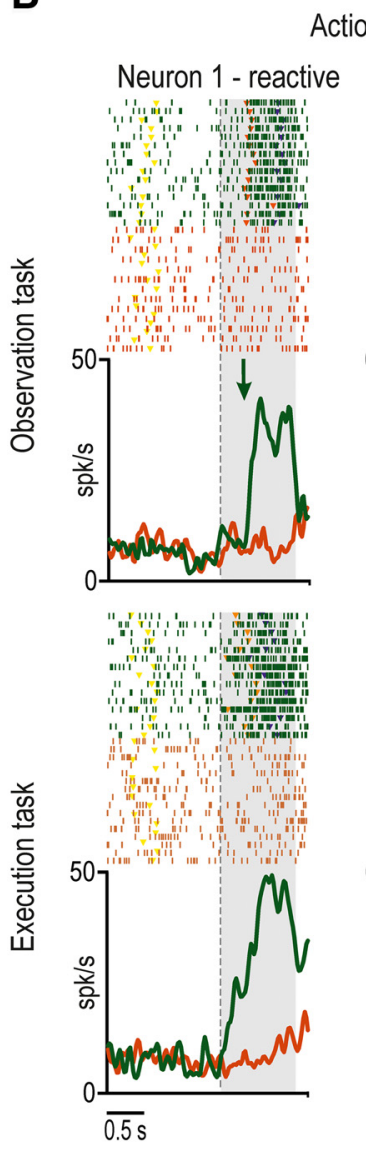

\section{Action MNs}

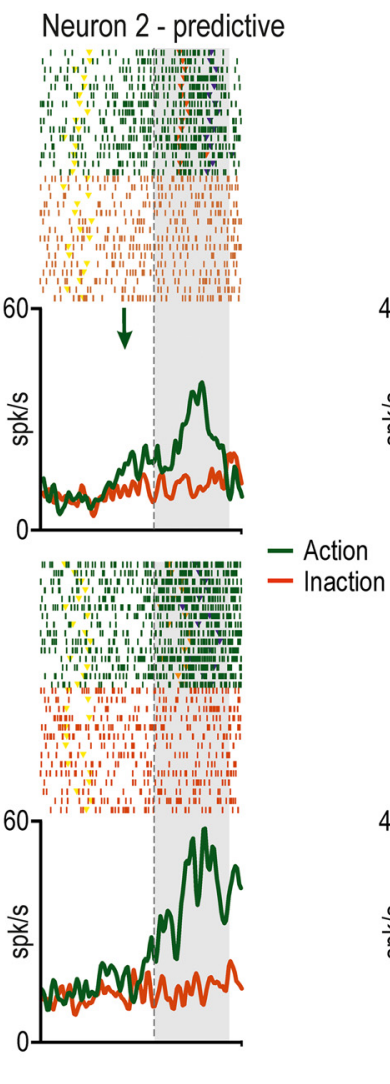

C

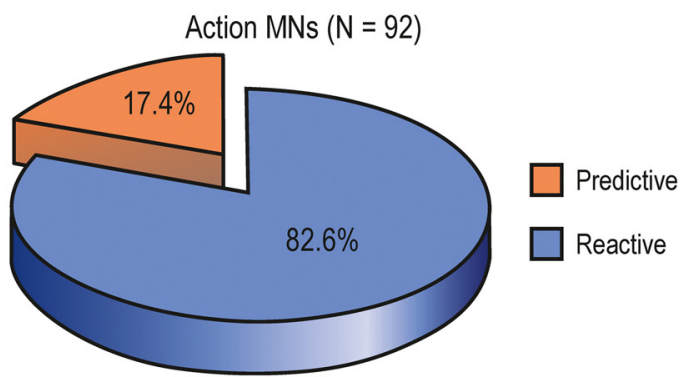

Inaction MNs

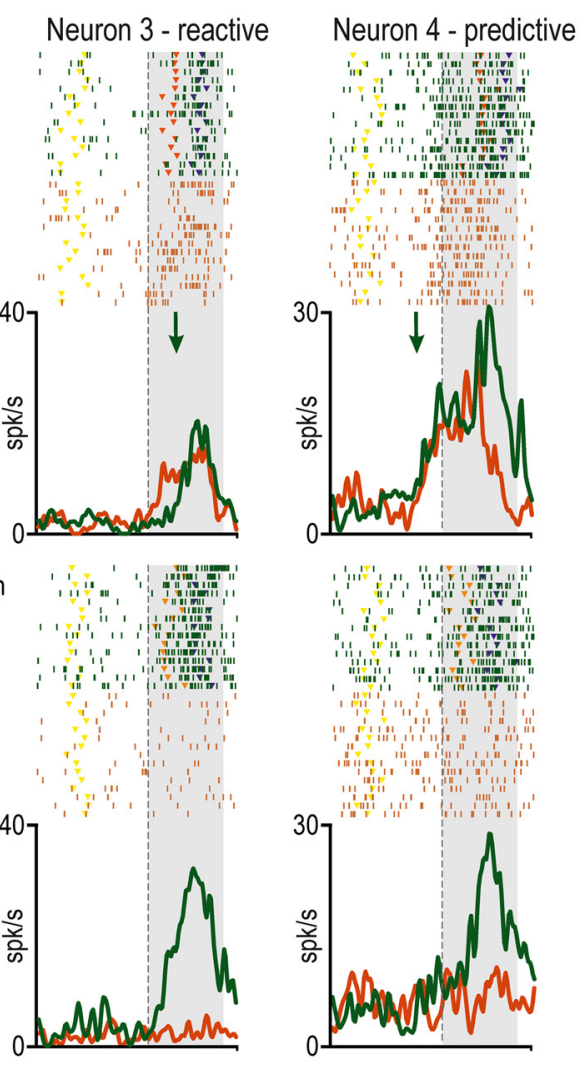

Figure 1. A, Phases of the tasks. Note that the temporal structure and task phases of the $0 T$ and $E T$ are identical. $B$, Examples of the visual and motor responses of Action and Inaction MNs showing reactive or predictive discharge during the $0 T$. Rasters and histograms are aligned on the cue sound offset (go/no-go signal, dashed vertical lines). The gray-shaded regions indicate the time windows used for the statistical analysis. Yellow, object presentation; orange, detachment of hand from the starting position (reaching onset); blue, object-pulling onset. Green arrows indicate the timing of discharge onset (see Materials and Methods for details on the procedure for the identification of discharge onset timing). C, Pie charts showing the percentage of Action and Inaction MNs with predictive or reactive discharge. 

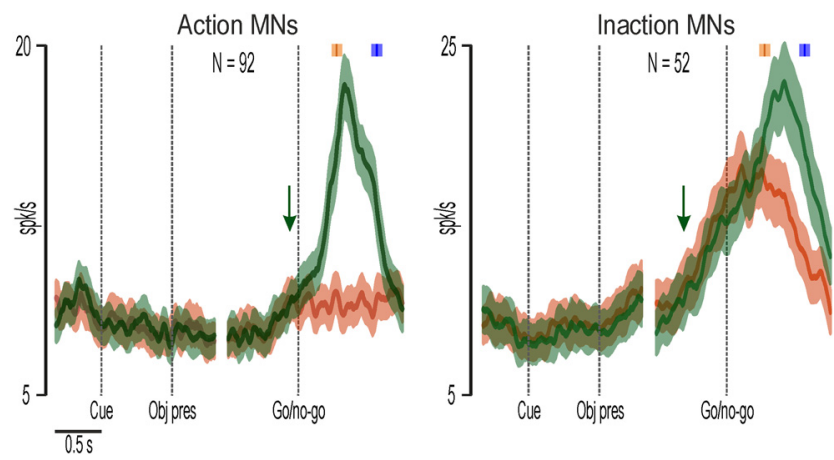

Figure 2. Population activity of Action and Inaction MNs during both Action (green) and Inaction (red) conditions of the $0 T$. In each part, the left side is aligned on the cue sound onset and object presentation (dashed vertical lines), while the right side is aligned on the go/no-go signal. The median time of reaching onset and object-pulling onset are indicated with the orange and blue markers, respectively, above each population plot. Shaded areas around each marker represent the 25 th and 75 th percentile times of other events of the same type. The green arrows indicate the onset timing of the action observation response. The green and red shadings around each curve represent $1 \mathrm{SE}$.

tested during observation of actions performed in the monkeys' extrapersonal rather than peripersonal space.

\section{Materials and Methods}

Experiments were performed on two male adult monkeys: M1 (Macaca nemestrina, $9 \mathrm{~kg}$ ) and M2 (Macaca mulatta, $7 \mathrm{~kg}$ ). Both animals were trained to perform the task described below with the hand (left) contralateral to the hemisphere to be recorded (right). After training completion, a head-fixation system and a recording chamber were implanted under general anesthesia (ketamine hydrochloride, $5 \mathrm{mg} / \mathrm{kg}$, i.m. and medetomidine hydrochloride, $0.1 \mathrm{mg} / \mathrm{kg}$, i.m.), followed by postsurgical pain medications. Surgical procedures were the same as previously described (Bonini et al., 2012). All the experimental protocols were approved by the Veterinarian Animal Care and Use Committee of the University of Parma and complied with the European law on the humane care and use of laboratory animals.

Apparatus and behavioral paradigm

Monkeys were trained to perform a go/no-go visuomotor task (Execution Task, ET) and, in a subsequent block of trials, to observe the experimenter (from a $90^{\circ}$ visual perspective) performing the same task (Observation Task, OT). The apparatus used for the tasks has been described in detail previously (Bonini et al., 2014a).

The tasks included two basic conditions ( 15 trials each one), randomly interleaved: Action (in which the monkey, or the experimenter, grasped a previously presented object) and Inaction (in which the monkey, or the experimenter, were required to remain still). All grasping neurons included in the present study also responded during grasping in the dark. Although three different objects were presented, here we focus on data obtained with a single object (a big metallic cone), because previous studies (Bonini et al., 2014a, b) showed that object selectivity was rare during the OT. Action and Inaction conditions were characterized by the same temporal sequence of events during both the ET and OT (Fig. 1A) as follows.

Action condition. A fixation point was presented, in complete darkness, and the monkey was required to start fixating it within $1.2 \mathrm{~s}$. Fixation onset determined the presentation of a cue sound (a $1200 \mathrm{~Hz}$ sine wavehigh tone) associated with Action condition. After $0.8 \mathrm{~s}$ the target object became visible. Then, after a variable time lag $(0.8-1.2 \mathrm{~s})$, the sound ceased (go signal), and the monkey had to reach, grasp, and pull (for at least $0.8 \mathrm{~s}$ ) the object. A fixed amount of juice was automatically delivered by a computer-controlled device (Crist Instruments) as a reward following correctly performed trials.

Inaction condition. The temporal sequence of events was the same as for Action condition, but a different cue sound (a $300 \mathrm{~Hz}$ sine wave-low tone) instructed the monkey to remain still until the end of the trial, maintaining fixation (for $1.2 \mathrm{~s}$ ) even after the end of the sound (no-go signal). If the monkey correctly performed the task, without moving or breaking fixation, the reward was automatically delivered.

In the OT, regardless of whether the experimenter was performing the Action or Inaction condition, only the experimenter's hand (not the whole body) was visible, and the monkey was required to remain still and to maintain fixation until the end of the trial to get the reward. The task phases were automatically controlled by LabView-based software. If the monkey broke fixation, made an incorrect movement, or did not respect the task constrains, the trial was aborted and no reward was delivered. In some sessions, MN activity was also studied while monkeys observed the experimenter performing the action within their peripersonal space (Bonini et al., 2014a).

\section{Recording techniques}

Neuronal recordings were performed by means of multielectrode linear arrays (16-channel U-probes, Plexon, and 16-channel silicon probes; Ruther et al., 2010; Herwik et al. 2011; distributed by Atlas Neuroengineering), with impedance (measured at $1 \mathrm{kHz}$ ) ranging from 0.3 to 1.5 $\mathrm{M} \Omega$. Devices and techniques used for probe insertions have been described previously (Bonini et al., 2013, 2014a).

The signal was amplified and sampled at $40 \mathrm{kHz}$ with a 16 -channel OmniPlex system (Plexon). All final quantitative analyses were performed off-line, as described hereafter.

Recording of behavioral events and definition of epochs of interest Contact-sensitive devices (Crist Instruments) were used to detect when the monkey or the experimenter touched the metal surface of the starting position or the target object with the hand. A switch located behind the object signaled the onset of the object-pulling phase. TTL signals were used by LabView-based software to monitor the monkey's performance and to control the presentation of auditory and visual cues of the behavioral paradigm as well as the reward delivery. All these signals were fed to the recording system to be stored in parallel with neuronal activity, to exploit them to align neuron activity, to construct the response histograms, and for statistical analysis.

Eye position was monitored with an eye-tracking system composed by a $50 \mathrm{~Hz}$ CCD video camera (Ganz; F11CH4) provided with an infrared filter and two spots of infrared light. Analog signal related to horizontal and vertical eye position was fed to a computer equipped with dedicated software (Pupil), enabling calibration and processing of eye position. The monkey was required to maintain its gaze on the fixation point (tolerance radius $5^{\circ}$ ) during all the tasks.

Different epochs of interest were defined: (1) baseline, $500 \mathrm{~ms}$ before object presentation; (2) object presentation, from 50 to $450 \mathrm{~ms}$ after switching on the light; (3) precue, $500 \mathrm{~ms}$ before sound off; and (4) postcue, from go/no-go signal to $1000 \mathrm{~ms}$ after this event. The same epochs were used to analyze neuronal responses during ET and OT.

Although it is well established that MN activation can be dissociated from muscle activation (Kraskov et al., 2014), we directly excluded that monkeys produced subtle hand or arm movements during action observation or inaction condition by recording EMG from the extensor digitorum communis and the deltoid of the body side contralateral to the recorded hemisphere. The findings (Bonini et al., 2014b) demonstrated the absence of muscle activity in both conditions.

\section{Data analyses}

The raw signals of all trials of a recording session were high-pass filtered $(300 \mathrm{~Hz})$, and single-unit action potentials were sorted off-line (Plexon) using principal component and template matching techniques, as previously described (Bonini et al., 2014a).

Preliminary analyses were performed to identify neurons with mirror properties. First, we applied a one-way repeated-measures ANOVA (four levels of the factor Epoch, $p<0.01$ ) to each neuron response in the Action condition of the ET and OT, separately. Neurons showing stronger discharge during the postcue epoch, relative to baseline and object presentation epochs in both ET and OT (Bonferroni post hoc, $p<0.05$ ), were classified as mirror neurons. Note that, in this way, the response can never be accounted for by simple object-related activity. The same ANOVA was also applied to study possible neuron response in the Inac- 
A

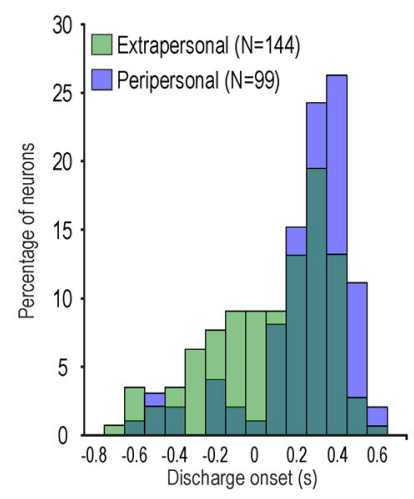

B

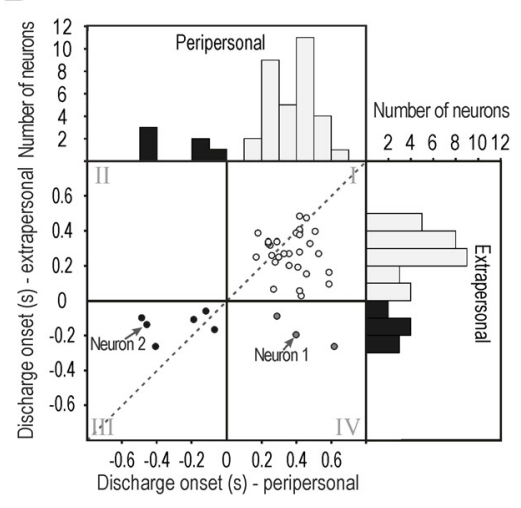

C

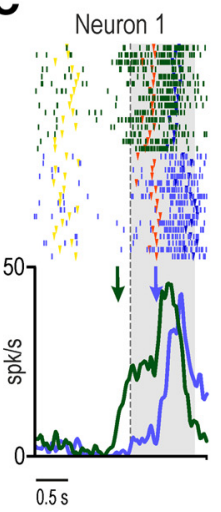

Neuron 2

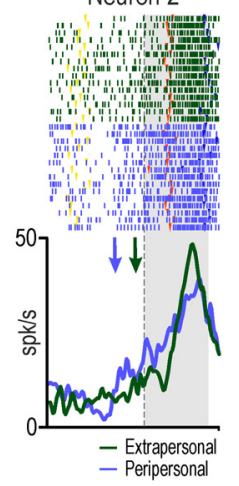

Figure 3. A, Distribution of the discharge onset timing, relative to the go-signal, of MNs discharging in the extrapersonal (green) or peripersonal (blue) space overlapped. $\boldsymbol{B}$, Scatter plot and histograms illustrating the distribution of discharge onset timing among MNs responding to the observation of actions in both the peripersonal and extrapersonal space. The dashed line indicates the function $x=y$. C, Examples of two MNs discharging during the observation of actions in the peripersonal and extrapersonal space. Green and blue arrows specify the timing of discharge onset in the extrapersonal and peripersonal space, respectively. Other conventions are as in Figure 1.

tion condition of ET and OT, separately. In particular, we looked for possible responses during precue and/or postcue epoch relative to both baseline and object presentation epochs (Bonferroni post hoc, $p<0.05$ ). All significant Inaction responses during the OT were then compared with possible Inaction responses during the ET (paired-sample $t$ test, $p<$ $0.05)$, to identify MNs specifically responding to Inaction during the OT. Each neuron was considered to be modulated in a specific task if it fired at least 5 spikes/s.

Based on these analyses, we classified MNs as Action or Inaction (Bonini et al., 2014b), depending on whether they discharged during Action condition only, or during Inaction condition as well, respectively. The discharge pattern of each MN was then classified as "predictive" or "reactive" relative to the go signal. This choice certainly implies an underestimation of the number of predictive neurons relative to what one could expect by using the experimenter's reaching onset as a reference. Nonetheless, it enables false positive results to be avoided, since handwrist movements start before actual reaching onset and they might trigger $\mathrm{MN}$ discharge. Each neuron firing rate, aligned on the go signal, was averaged on a trial-by-trial basis $(N=15)$ in a time range of $\pm 0.8 \mathrm{~s}$ relative to the alignment point, using $200 \mathrm{~ms}$ bins slid forward in steps of $10 \mathrm{~ms}(N=142$ bins $)$. The average firing rate in each bin was then compared with that during object presentation epoch (paired-sample $t$ test). The timestamp corresponding to the first of a series of (at least) seven consecutive bins with an uncorrected $p$ value lower than 0.05 was taken as the discharge onset time. Neurons with discharge onset timing earlier than the go signal (negative values) were classified as predictive, while those with positive values were classified as reactive. This further criterion lead to a total of four different discharge patterns: Action MNs with reactive or predictive discharge and Inaction MNs with reactive or predictive discharge.

\section{Results}

We recorded 205 MNs from area F5: 89 were studied only with the OT performed in the extrapersonal space, while 116 were tested both in the extrapersonal and peripersonal space. We will first describe the discharge pattern of MNs during the observation of actions in the monkeys' extrapersonal space, and then we will compare it with that obtained during the observation of actions in the peripersonal space.

\section{Predictive coding of others' actions observed in the extrapersonal space}

The MNs responding during the OT performed in the extrapersonal space $(N=144)$ were subdivided into two main types (see Materials and Methods): (1) classical Action MNs (Fig. 1B, neurons 1 and 2), specifically discharged during action observation (green), but not during the Inaction condition (red), and (2) Inaction MNs (Fig. 1B, neurons 3 and 4; Bonini et al., 2014b), discharged not only during action observation, but also during the Inaction condition of the OT, but not of the ET.

Each $\mathrm{MN}$ was then analyzed to establish whether the onset of its visual response (Fig. $1 B$, green arrows) was predictive or reactive. Neurons 1 and 3 (Fig. $1 B$ ) became active only after the go signal, in tight relationship with the experimenter's movement onset (orange triangles in the raster plot), thus showing reactive discharge. In contrast, neurons 2 and 4 started firing well in advance of the go signal, when the experimenter's hand was still, thus exhibiting predictive discharge. Interestingly, most of Inaction MNs (31/52) showed predictive discharge, while only a smaller fraction (16/92) of Action MNs showed it $\left(\chi^{2}=26.94\right.$, $p<0.001$; Fig. $1 C$ ). These findings suggest that predictive discharge is more frequently associated with Inaction MNs, while Action MNs show more often reactive discharge.

To test the robustness of these latter associations, we performed two distinct population analyses. Figure 2 shows the response of Action and Inaction MNs with increased activity during action observation, pooled together regardless of their pattern of discharge (reactive or predictive). It is clear that both neuronal populations increased their activity before the go signal (green arrows), although Inaction MNs became active earlier $(-480 \mathrm{~ms})$ as compared with Action MNs ( $-100 \mathrm{~ms})$.

By considering all the recorded MNs together, we found that the average onset timing of the population activity relative to the go signal was $-340 \mathrm{~ms}$; that is, hundreds of milliseconds before the timing of $\mathrm{MN}$ visual response reported in previous studies ( $+60 \mathrm{~ms}$ relative to the onset of the agent's reaching movement; Maranesi et al., 2013), in which monkeys were not provided with any auditory or visual cue enabling them to predict the onset of the observed actions.

\section{Space dependency of $\mathrm{MN}$ predictive and reactive coding of others' observed actions}

It is known that MNs can discharge differently depending on the space in which an observed action occurs (Caggiano et al., 2009), suggesting that, in addition to action understanding, MNs might be involved in preparing appropriate motor responses during social interactions. Since predictive discharge might be crucial for this latter function, we investigated whether MN discharge pattern is related to the space in which the observed action is per- 
formed. To this purpose, in some penetrations we studied neuron activity during action observation in the peripersonal space.

Among the $116 \mathrm{MNs}$ tested in the peripersonal space (32 recorded from M1 and 84 from M2), 99 responded in this latter condition. It is noteworthy that only $11(11.1 \%)$ of them showed predictive discharge, while $47 / 144(32.6 \%)$ of those responding in the extrapersonal space showed this behavior $\left(\chi^{2}=14.96, p<\right.$ 0.001 ). In addition, Mann-Whitney test indicated that MN responses studied in the extrapersonal space started significantly earlier (median $200 \mathrm{~ms}$ ) than those in the peripersonal space (median $360 \mathrm{~ms}, Z=4.96, p<0.001$; Fig. $3 A$ ). These findings indicate that the representation of others' actions is activated earlier when the observed agent acts in the extrapersonal rather than peripersonal space.

This finding is also supported by the analyses performed on a subset of MNs responding to the observation of actions both when they were performed in the peripersonal and extrapersonal space $(38 / 99,19$ recorded from M1 and 19 from M2). Figure $3 B$ shows the distribution of the discharge onset timing of all these neurons, in both space sectors. Although there is no significant correlation between discharge onset time in the peripersonal and extrapersonal space $(r=0.28, p=0.081)$, it is clear that all but three MNs maintained their predictive or reactive discharge pattern when tested in different spaces (Fig. 3B, white and black dots in scatter plot). However, even in this subset of MNs, discharge onset was earlier when the monkey observed the action performed in its extrapersonal (median $0.26 \mathrm{~s}$ ) rather than peripersonal (median $0.36 \mathrm{~s}$ ) space (Wilcoxon paired-samples test: $Z=1.97, p=0.04$; Fig. $3 C$, neuron 1 ). Nevertheless, it is also interesting to note that five MNs with predictive discharge in the extrapersonal space (13.2\%) showed an even earlier discharge onset when they were tested in the peripersonal space (Fig. 3B, black dots in the third quadrant, $C$, neuron 2).

\section{Discussion}

In this study, we provided the first demonstration that MN discharge can largely anticipate the onset of observed actions within a predictable context. At the population level, a striking contrast emerges between these findings and previous studies in which MNs were recorded in unpredictable contexts (Maranesi et al., 2013): indeed, while in this latter work the MN population response was reactive relative to the experimenter's movement onset $(+60 \mathrm{~ms})$, here we showed that MN population activity was largely predictive $(-340 \mathrm{~ms})$, even relative to the go signal, which occurred before movement onset. Thus, the presence of contextual information that the monkey is able to use for acting enables MNs to predict, rather than react to, others' observed actions.

These results support, at the single neuron level, previous human studies based on different indirect techniques (Kilner et al., 2004; Aglioti et al., 2008; Southgate et al., 2009), showing that the $\mathrm{MN}$ system does not merely recognize others' actions through forward sensorimotor-matching mechanisms, but also predicts them ahead of their unfolding. Predictive discharge is tightly associated with the capacity of MNs to represent potential actions at a high level of abstraction. Indeed, while the great majority of Action MNs showed reactive discharge, most of Inaction MNs, which encode representation of an action not only during its execution and observation but also during others' inactions (Bonini et al., 2014b), showed largely predictive activation. Interestingly, the predictive activation during action and inaction conditions is initially similar before the go/no-go signal, but then diverges, becoming stronger during overt action observation relative to the inaction condition (Fig. 2). This finding supports the idea that the discharge of Inaction MNs generates an internal, predictive representation of others' actions, which can then be strengthened when visual information is provided.

These findings fit well with the predictive coding account of the MN system (Kilner et al., 2007), but the mechanism suggested by our single neuron data appears more complex. Indeed, both sensory-driven (forward model) and internally generated (generative model) representations of the same observed action seem to be recruited in a predictable context: the former are mainly based on Action MN activity, the latter mostly rely on the predictive activation of Inaction MNs. This proposal fits with the available anatomical data showing that, in area F5, there is a convergence of information from the superior temporal sulcus, through the inferior parietal cortex, concerning others' observed actions, and from the higher order prefronto-dependent motor (Luppino et al., 1993; Gerbella et al., 2011) and prefrontal (Borra et al., 2011; Gerbella et al., 2013) areas. Thus, the MN system can play a role both in sensory-driven action representation, through the parietofrontal sensorimotor pathway, and context-based action prediction, through the prefronto-premotor routes, although these processes appear to rely on partially distinct neuronal populations.

What might be the advantage of having distinct neuronal populations dedicated to predictive and reactive coding of others' actions? Some intriguing explanations might be derived from our finding that, at least for some MNs, the pattern of discharge can change, from predictive to reactive or vice versa, depending on the space in which the observed action occurred. Different sets of MNs could show predictive discharge in the extrapersonal or peripersonal space to specifically prepare space-constrained behaviors during social interactions (Caggiano et al., 2009). For example, motor prediction in the peripersonal space could underlie motor reactions during competitive or defensive situations, while predicting others' actions in the extrapersonal space might induce one to prepare approaching behaviors. The earlier activation observed when MNs were tested in the extrapersonal relative to the peripersonal space suggests that preparing for behavioral responses to others' actions occurring in the far space requires more time, thus earlier prediction of others' upcoming movements. Note that, in addition to the action goal, $\mathrm{MN}$ discharge could encode predictive representations of many specific action features, such as direction, view point, or grip type, but further studies are needed to clarify this issue and the possible relevance of predictive and reactive coding of others' actions during different forms of social interaction.

\section{References}

Aglioti SM, Cesari P, Romani M, Urgesi C (2008) Action anticipation and motor resonance in elite basketball players. Nat Neurosci 11:1109-1116. CrossRef Medline

Bonini L, Ugolotti Serventi F, Bruni S, Maranesi M, Bimbi M, Simone L, Rozzi S, Ferrari PF, Fogassi L (2012) Selectivity for grip type and action goal in macaque inferior parietal and ventral premotor grasping neurons. J Neurophysiol 108:1607-1619. CrossRef Medline

Bonini L, Maranesi M, Livi A, Bruni S, Fogassi L, Holzhammer T, Paul O, Ruther P (2013) Application of floating silicon-based linear multielectrode arrays for acute recording of single neuron activity in awake behaving monkeys. Biomed Tech. Advance online publication. Retrieved Oct. 26, 2013. doi: 10.1515/bmt-2012-0099.

Bonini L, Maranesi M, Livi A, Fogassi L, Rizzolatti G (2014a) Spacedependent representation of objects and other's action in monkey ventral premotor grasping neurons. J Neurosci 34:4108-4119. CrossRef Medline

Bonini L, Maranesi M, Livi A, Fogassi L, Rizzolatti G (2014b) Ventral premotor neurons encoding representations of action during self and others' inaction. Curr Biol 24:1611-1614. CrossRef Medline 
Borra E, Gerbella M, Rozzi S, Luppino G (2011) Anatomical evidence for the involvement of the macaque ventrolateral prefrontal area $12 \mathrm{r}$ in controlling goal-directed actions. J Neurosci 31:12351-12363. CrossRef Medline

Caggiano V, Fogassi L, Rizzolatti G, Thier P, Casile A (2009) Mirror neurons differentially encode the peripersonal and extrapersonal space of monkeys. Science 324:403-406. CrossRef Medline

Fogassi L, Ferrari PF, Gesierich B, Rozzi S, Chersi F, Rizzolatti G (2005) Parietal lobe: from action organization to intention understanding. Science 308:662-667. CrossRef Medline

Gallese V, Fadiga L, Fogassi L, Rizzolatti G (1996) Action recognition in the premotor cortex. Brain 119:593-609. CrossRef Medline

Gerbella M, Belmalih A, Borra E, Rozzi S, Luppino G (2011) Cortical connections of the anterior (F5a) subdivision of the macaque ventral premotor area F5. Brain Struct Funct 216:43-65. CrossRef Medline

Gerbella M, Borra E, Tonelli S, Rozzi S, Luppino G (2013) Connectional heterogeneity of the ventral part of the macaque area 46 . Cereb Cortex 23:967-987. CrossRef Medline

Herwik S, Paul O, Ruther P (2011) Ultrathin silicon chips of arbitrary shape by etching before grinding. J Microelectromech Syst 20:791-793. CrossRef

Kilner JM, Vargas C, Duval S, Blakemore SJ, Sirigu A (2004) Motor activation prior to observation of a predicted movement. Nat Neurosci 7:12991301. CrossRef Medline

Kilner JM, Friston KJ, Frith CD (2007) Predictive coding: an account of the mirror neuron system. Cogn Process 8:159-166. CrossRef Medline
Kohler E, Keysers C, Umiltà MA, Fogassi L, Gallese V, Rizzolatti G (2002) Hearing sounds, understanding actions: action representation in mirror neurons. Science 297:846-848. CrossRef Medline

Kraskov A, Philipp R, Waldert S, Vigneswaran G, Quallo MM, Lemon RN (2014) Corticospinal mirror neurons. Philos Trans R Soc Lond B Biol Sci 369:20130174. CrossRef Medline

Luppino G, Matelli M, Camarda R, Rizzolatti G (1993) Corticocortical connections of area F3 (SMA-proper) and area F6 (pre-SMA) in the macaque monkey. J Comp Neurol 338:114-140. CrossRef Medline

Maranesi M, Ugolotti Serventi F, Bruni S, Bimbi M, Fogassi L, Bonini L (2013) Monkey gaze behaviour during action observation and its relationship to mirror neuron activity. Eur J Neurosci 38:3721-3730. CrossRef Medline

Rizzolatti G, Fadiga L, Gallese V, Fogassi L (1996) Premotor cortex and the recognition of motor actions. Brain Res Cogn Brain Res 3:131-141. CrossRef Medline

Ruther P, Herwik S, Kisban S, Seidl K, Paul O (2010) Recent progress in neural probes using silicon MEMS technology. IEEJ Trans Elec Electron Eng 5:505-515. CrossRef

Southgate V, Johnson MH, Osborne T, Csibra G (2009) Predictive motor activation during action observation in human infants. Biol Lett 5:769_ 772. CrossRef Medline

Umiltà MA, Kohler E, Gallese V, Fogassi L, Fadiga L, Keysers C, Rizzolatti G (2001) I know what you are doing. a neurophysiological study. Neuron 31:155-165. CrossRef Medline 